\title{
JMSR
}

Journal of Medical and Scientific Research

\section{Clinico-epidemiological profile of SARS-CoV-2 infection in individuals investigated at tertiary care hospital}

\author{
Nikita Sherwani ${ }^{1}$, Neha Singh ${ }^{1, *}$, Nirmal Verma ${ }^{2}$, Kamlesh Jain ${ }^{2}$, Arvind Neral $^{3}$, Rupam Gahlot ${ }^{1}$, Tripti Dhurandhar ${ }^{2}$, \\ and Harsh Deep \\ ${ }^{1}$ Virology Lab, Department of Microbiology, Pt. JNM Medical College, Raipur, Chhattisgarh-492001, India \\ ${ }^{2}$ Department of Community Medicine, Pt. JNM Medical College, Raipur, Chhattisgarh-492001, India \\ ${ }^{3}$ Department of Pathology, Pt. JNM Medical College, Raipur, Chhattisgarh-492001, India
}

\begin{abstract}
Background: The World Health Organization (WHO) declared the pandemic disease caused by SARS-CoV-2 as coronavirus disease 2019 (COVID-19). Presently, COVID-19 has infected individuals in over seventy nations across the world. China had a significant COVID-19 impact and taken great measures to restrict the disease's development and increase the cure rate.

Objective: Research paper describes the clinico-epidemiological profile of SARS-CoV-2 infection in individuals tested at state key Virology Research and Diagnostic Laboratory (VRDL), Pt. J.N.M. Medical College, Raipur (CG), India.

Method: Respiratory samples oropharyngeal and nasopharyngeal swabs were collected in VTM from suspected COVID-19 individuals for laboratory analysis and cases were confirmed by reverse transcription polymerase chain reaction (RT-PCR). A total of 32,145 patients with confirmed diagnosis of SARS-CoV-2 infection were included in this study. Data included in this study, from the period of August 2020 to February 2021, were collected from offline and online medical records.

Result: Amongst 32,145 SARS-CoV-2 infected patients, 19674 (61\%) were males and 12471 (39\%) were females, $5756(17.90 \%)$ were symptomatic and 26,389 (82.10\%) were asymptomatic. Maximum percentage of symptomatic subjects were found in 70-79 years (25\%) followed by 60-69 years (24\%), out of 5756 symptomatic patients $3772(65.53 \%)$ were males and $1984(34.46 \%)$ were females, most common presenting symptom was cough $(80.9 \%)$ followed by fever $(33.8 \%)$, sore throat $(16.1 \%)$ breathlessness $(14.1 \%)$. The most common comorbidity found among symptomatic group was hypertension followed by diabetes.
\end{abstract}

Conclusion: COVID-19 is an evolving disease and data from our study help in understanding the clinicepidemiological profile of patients.

Keywords: SARS-CoV-2; clinico-epidemiological; RT-PCR; COVID-19

*Corresponding author: Dr. NehaSingh, Virology lab, Department of Microbiology, Pt. JNM Medical College, Raipur, Chhattisgarh492001, India.Email: nevi0007@gmail.com

Received 18 June 2021; Revised 2 September 2021; Accepted 14 September 2021; Published 22 September 2021

Citation: Sherwani N, Singh N, Verma N, Jain K, Neral A, Gahlot R, Dhurandhar T, Deep H. Clinico-epidemiological profile of SARS-CoV-2 infection in individuals investigated at tertiary care hospital. J Med Sci Res. 2021; 9(4):187-191. DOI: http://dx.doi. org/10.17727/JMSR.2021/9-28

Copyright: (C) 2021 Sherwani N et al. Published by KIMS Foundation and Research Center. This is an open-access article distributed under the terms of the Creative Commons Attribution License, which permits unrestricted use, distribution, and reproduction in any medium, provided the original author and source are credited. 


\section{Introduction}

In late December 2019, an outbreak of an emerging disease (COVID-19) due to a novel coronavirus (named SARS-CoV-2 later) started in Wuhan, China and rapidly spread in China and out countries. After the declaration of COVID-19 infection as pandemic by World Health Organization (WHO) on $11^{\text {th }}$ March 2020, there is rise in the cases of COVID-19 worldwide as well as in India [1]. All populations are susceptible to SARS-CoV-2 particular attention and efforts to protect or reduce transmission should be directed at vulnerable groups such as children, health care providers, pregnant women, and the elderly. Analysis on family clusters and an assessment of the basic reproductive number based on early investigation data have shown efficient person-to-person transmissibility of the virus [2]. Nevertheless, there is a need to verify and update these primary findings as the number of patients accumulates. Viral Research and Diagnostic Laboratory (VRDL), Department of Microbiology, Pt. J.N.M. Medical College, Raipur is one of the key testing centres in Chhattisgarh where the samples are subjected to reverse transcription polymerase chain reaction (RT-PCR) for testing of COVID-19 infection. Therefore, a cross-sectional observational study has been proposed to find out the clinicoepidemiological profile of SARS-CoV-2 infection in individuals tested to acquire knowledge regarding the virus and employ it in making strategies and providing specific interventions for prevention and management.

\section{Material and methods}

From the period of August 2020 to February 2021, a cross-sectional observational study was conducted on patients who presented for COVID-19 screening at our virology laboratory. Consecutive sampling technique was used, all individuals who had given a sample for evaluation and who appeared to the hospital with respiratory symptoms or signs of SARS$\mathrm{CoV}-2$ infection or were in contact with lab confirmed SARS-CoV-2 infected patients or were hospital employees or had international travel history in the previous 14 days were included in the study group. Specimen referral forms (SRF) included information regarding recent exposure history, travel history, clinical symptoms, comorbidities, and demographic details. During the collection and processing of samples, all healthcare and laboratory personnel wore personal protective equipment (PPE). Samples from the nasopharynxand oropharynx were collected in viral transport media (VTM, HiViral ${ }^{\mathrm{TM}}$ ) and Viral RNA was extracted using spin column-based kits (HiMedia) [3-4]. RT-PCR test was performed by using an FDA-approved and recommended kit (SD Biosensor, Korea), which was designed according to WHO interim guidance for laboratory RT-PCR testing for SARS-CoV-2. The kit is designed to target the rdrp gene for confirmatory test and $\mathrm{E}$ gene for screening. All RT-PCR assessments were competed using the LightCycler $^{\circledR} 96$ Instrument (Roche) platform. Categorical variables such as sex, age, comorbidities, or any symptoms were expressed in terms of frequency and the differences between groups symptomatic and asymptomatic patients were compared.

\section{Results}

\section{Demographic characteristics}

Over a period from August 2020 to February 2021, a total of 32,145 laboratory confirmed COVID19 positive patients were detected. Demographic analysis revealed that majority of these patients were males [males $61 \%(n=19,674)$ vs. females $39 \%$ $(\mathrm{n}=12,471)]$. Most of the patients belonged to age group 20-29 years $(\mathrm{n}=7476,23.25 \%)$ followed by age group 30-39 ( $\mathrm{n}=7389,22.98 \%)$. A few patients were observed in $>90(\mathrm{n}=21,0.06 \%)$ years of age group (Table 1).

Table 1: Demographic distribution in patients with COVID-19.

\begin{tabular}{|cc|}
\hline & Frequency (\%) \\
\hline Gender $(\mathrm{n}=32,145)$ & $19,674(61 \%)$ \\
Fale & $12,471(39 \%)$ \\
Age group (years) & \\
$0-9$ & $1336(4.15 \%)$ \\
$10-19$ & $2751(8.55 \%)$ \\
$20-29$ & $7476(23.25 \%)$ \\
$30-39$ & $7389(22.98 \%)$ \\
$40-49$ & $5316(16.53 \%)$ \\
$50-59$ & $4354(13.54 \%)$ \\
$60-69$ & $2413(7.50 \%)$ \\
$70-79$ & $871(2.70 \%)$ \\
$80-89$ & $205(0.63 \%)$ \\
$>90$ & $21(0.06 \%)$ \\
\hline
\end{tabular}




\section{Distribution of symptomatic and asymptomatic subjects according to age groups}

Amongst 32,145 COVID-19 positive patients' percentage of symptomatic and asymptomatic COVID positive subjects varies in different age groups. Maximum percentage of symptomatic COVID positive subjects were found in 70-79 years (25\%) followed by $60-69$ years (24\%), $50-59$ years $(22 \%)$, 40-49 years $(19 \%)$ whereas minimum percentage of symptomatic COVID positive subjects were found in 0 -9 years $(10 \%)$ followed by $10-19$ years $(12 \%)$ (Figure 1). Males $(n=3772,65.53 \%)$ were more in number in symptomatic group as compared with females symptomatic $(\mathrm{n}=1984,34.47 \%)$ and comparison of percentage of symptomatic COVID19 positive subjects in various age groups between males and females. The percentage of symptomatic COVID-19 positive subjects are more in females than males in only two age groups (0-9 years and 80-89 years) whereas in rest all the age groups, percentage of symptomatic COVID-19 positive subjects are more in males than females (Figure 2).

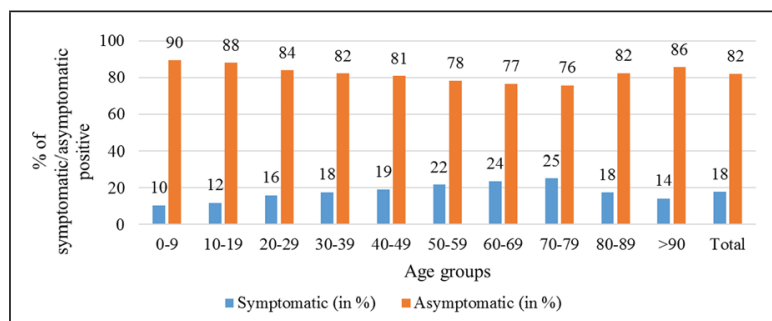

Figure 1: Distribution of percentages of symptomatic and asymptomatic COVID-19 positive subjects according to specific age groups.

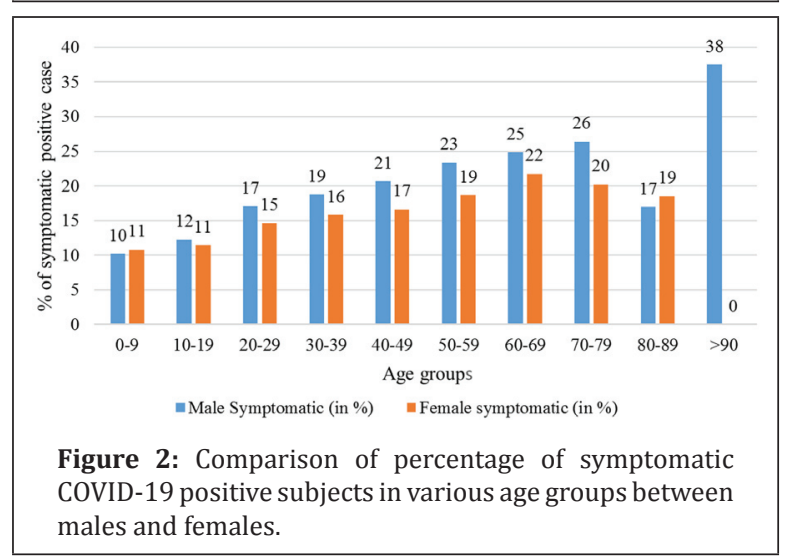

Clinical presentations of COVID-19 positive
symptomatic patients Cough $(80.9 \%)$ was the most common presentation of symptomatic positive patients followed by fever $(33.8 \%)$, sore throat (16.1\%), breathlessness (14.1\%), nasal discharge (10.8), body ache (9\%), vomiting (3.7\%), chest pain $(2.2 \%)$, haemoptysis $(1.2 \%)$, nausea $(1.2 \%)$, whereas least common presentation was abdominal pain $(0.9 \%)$ and diarrhoea (0.5\%) (Table 2).

Table 2: Clinical presentations of COVID-19 positive symptomatic patients.

\begin{tabular}{|lc|}
\hline Clinical symptoms & Frequency (\%) \\
\hline Cough & $4659(80.9 \%)$ \\
Fever at evaluation & $1943(33.8 \%)$ \\
Sore throat & $928(16.1 \%)$ \\
Breathlessness & $813(14.1 \%)$ \\
Body ache & $520(9.0 \%)$ \\
Nasal discharge & $624(10.8 \%)$ \\
Vomiting & $214(3.7 \%)$ \\
Chest pain & $124(2.2 \%)$ \\
Haemoptysis & $67(1.2 \%)$ \\
Nausea & $67(1.2 \%)$ \\
Abdominal pain & $52(0.9 \%)$ \\
Diarrhoea & $31(0.5 \%)$ \\
\hline
\end{tabular}

\section{Distribution of COVID-19 positive patients according to the pre-existing medical conditions}

Diabetes and hypertension constitute most common pre-existing medical conditions both in symptomatic and asymptomatic positive patients followed by malignancy and cardiac diseases (Figure 3).

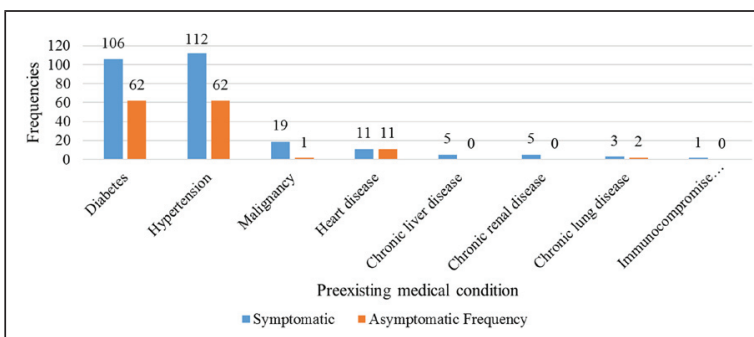

Figure 3: Distribution of COVID-19 positive patients according to the pre-existing medical conditions.

\section{Discussion}

The present study analyses the demographic characteristics and clinical presentation at the time of laboratory testing of 32,145 SARS-CoV-2 infected 
patients from VRDL, Pt. J.N.M. Medical College, Raipur. This study reports a smaller number of females which is in line with the findings from China and United States where the number of females infected were also less than $40 \%$ [5]. The lower proportion of women could be because majority of the working population in our country is males therefore, the chances of contact with infected person is more in male sex [6-7]. However, along with the social factors, genetic, hormonal difference, and immunological factors also play important role in the gender disparity. A recent study shows that the difference between the sex could be because of difference in the levels of angiotensin-converting enzyme-2 (ACE-2) expression, the first point of contact for SARS-CoV-2 and the human body, in both men and women [8-9]. ACE-2 gene locus is on $\mathrm{X}$-chromosome, which makes women heterozygous and differently assorted as compared to men who are homozygous. Along with this, testosterone also play role in the expression of ACE-2. An encouraging association between ACE-2 and coronavirus have been observed, different studies quantified the expression of ACE 2 proteins in human cells based on gender linked, for example, in studying the expression level and pattern of human ACE- 2 using a single-cell RNA-sequencing (RNA-seq), analysis showed that Asian males had higher expression of ACE-2 than female [10-11]. In general, testosterone plays inhibitory roles in immune processes in contrary to oestrogen, which is a possible explanation for men higher susceptibility to infections [12]. The majority of the cases belong to 20-39 years of age. Children and adolescent were less infected. In previous research studies suggests that children did not appear susceptible, or they could not catch infection easily. They were found to have relatively mild symptoms. There are many factors protecting children against SARS CoV-2 infection. One of the possible reasons could be due to strong immune response which is secondary to live vaccinations and frequent viral infections. Children have good lung regeneration capacity which contributing to early recovery. Along with this, there is absence of highrisk factors, like ageing related medical conditions, comorbidities and less degree of obesity, smoking as compared with adult population [13]. In this study, various symptoms reported among which, the most common symptom was cough followed by fever, sore throat, breathlessness and body ache. As reported by other studies, diabetes mellitus and hypertension were the most common comorbidities in our study. In other countries, diabetes mellitus has emerged as a most common comorbidities associated with severe disease [14-16]. Patients of COVID-19 with diabetes, are likely to experience hypoxia and will need ventilation control in intensive care unit (ICU) patients as compared with patients without diabetes. Similarly, diabetes, hypertension has also been linked to a significantly higher risk of respiratory infection [17-19].

\section{Conclusion}

Outbreaks of SARS-CoV and MERS-CoV in 2003 and 2012 respectively, coronaviruses were thought to cause mild, self-limiting respiratory infections in humans. Outbreak of SARS-CoV-2 pretending a massive challenge to global health, which will possibly run a prolonged way till herd immunity is achieved or an effective vaccine is found. Information generated from such studies will help in understanding the clinic-epidemiological profile of patients as well as in separating individuals with suspected symptoms which will be critical once the cases come down. Currently the high number of asymptomatic patients in our study is also as cause of concern as they can act as silent super spreaders.

\section{Recommendation}

Multicentric study should be conducted up to three to five years to get better understating and the pattern of this newly emerging disease.

\section{Limitation}

Single centre study.

\section{Conflicts of interest}

The authors declare no conflicts of interest.

\section{References}

[1] Siordia JA. Epidemiology and clinical features of COVID-19: A review of current Literature. J Clin Virol. 2020; 127:10431057.

[2] Wang L, Didelot X, Yang J, Wong G, Shi Y, et al. Inference of person-to-person transmission of COVID-19 reveals hidden super-spreading events during the early outbreak phase. Nat Communed. 2020; 11:5006.

[3] Bhandari S, Bhargava A, Sharma S, Keshwani P, Sharma R, et al. Clinical profile of COVID-19 infected patients admitted in a tertiary care hospital in north India. J Assoc Physicians India. 2020; 68(5):13-17.

[4] Liu L, Lei X, Xiao X, Yang J, Li J, et al. Epidemiological and Clinical Characteristics of Patients with Coronavirus Disease2019 in Shiyan City, China. Front Cell Infect Microbiol. 2020; $10: 284$. 
[5] Roger VL. Outcome's research and epidemiology: the synergy between public health and clinical practice. Circ Cardiovasc Qual Outcomes. 2011; 4(3):257-259.

[6] Zhong ZF, Huang J, Yang X, Peng JL, Zhang XY, et al. Epidemiological and clinical characteristics of COVID-19 patients in hengyang, hunan province, China. World J Clin Cases. 2020; 8(12):2554-2565.

[7] ICMR COVID study group, COVID epidemiology \& data management team, COVID laboratory team, VRDLN Team. Laboratory surveillance for SARS-CoV-2 in India: performance of testing \& descriptive epidemiology of detected COVID-19. Ind J Med Res 2020; 151(5):424.

[8] Dana PM, Sadoughi F. Hallajzadeh J, Asemi Z, Mansournia MA, et al. An insight into the sex differences in COVID-19 patients: what are the possible causes. Prehosp Disaster Med. 2020; 35(4):438-441.

[9] Liu J, Ji H, Zheng W, Wu X, Zhu JJ, et al. Sex differences in renal angiotensin converting enzyme 2 (ACE2) activity are $17 \beta$ oestradiol-dependent and sex chromosome-independent. Biol Sex Differ. 2010; 1(1):6.

[10] Zhonghua L, Xing B, Za ZX. Epidemiology working group for NCIP epidemic response, Chinese center for disease control and prevention. The epidemiological characteristics of an outbreak of 2019 novel coronavirus diseases (COVID-19) in China. 2020; 41(2):145-151.

[11] Shim E, Tariq A, Choi W, Lee Y, Chowell G. Transmission potential and severity of COVID-19 in South Korea. Int J Infect Dis. 2020; 93:339-344.

[12] Dhochak N, Singhal T, Kabra SK, Lodha R. Pathophysiology of COVID-19: Why Children fare better than adults? Indian J Pediatr. 2020; 87(7):537-546.

[13] Bunyavanich S, Do A, Vicencio A. Nasal gene expression of angiotensin-converting enzyme 2 in children and adults. JAMA. 2020; (23):2427-2429.

[14] Singh AK, Gupta R, Misra A. Comorbidities in COVID-19: Outcomes in hypertensive cohort and controversies with renin angiotensin system blockers. Diabetes Metab Syndr. 2020; 14(4):283-287.

[15] Scheen AJ, Marre M, Thivolet C. Prognostic factors in patients with diabetes hospitalized for COVID-19: Findings from the CORONADO study and other recent reports. Diabetes Metab. 2020; 46(4):265-271.

[16] Ip A, Parikh K, Parrillo JE, Mathura S, Hansen E, et al. Hypertension and renin-angiotensin- aldosterone system inhibitors in patients with COVID-19. MedRxiv. 2020; 1-10.

[17] Wu J, Mafham M, Mamas MA, Rashid M, Kontopantelis E, et al. Place and Underlying Cause of Death During the COVID19 Pandemic: Retrospective Cohort Study of 3.5 Million Deaths in England and Wales, 2014 to 2020. Mayo Clin Proc. 2021; 96(4):952-963.

[18] Wu C, Chen X, Cai Y, Xia J, Zhou X, et al. Risk factors associated with acute respiratory distress syndrome and death in patients with coronavirus disease 2019 pneumonia in Wuhan, China. JAMA Intern. Med. 2020; 180(7):934-943.

[19] Fehr AR, Perlman S. Coronaviruses: an overview of their replication and pathogenesis. Methods Mol Biol. 2015; 1282:1-23. 